\title{
MATCHED-FIELD SOURCE LOCALIZATION WITH NON-SYNCHRONIZED SENSOR ARRAYS
}

\author{
Stan E. Dosso \\ School of Earth and Ocean Sciences, University of Victoria \\ Victoria B.C. Canada V8W 3P6
}

\section{Overview}

This paper considers combining information from multiple hydrophone arrays in matched-field localization for an acoustic source in the ocean. Assuming each individual array is comprised of calibrated sensors which are synchronized in time, conventional matched-field methods can be applied for each array using the Bartlett (linear) processor and the resulting processors summed over arrays. However, if the relative calibration and/or time synchronization is known between some or all arrays, more informative multiple-array processors can be derived by maximum-likelihood methods. For example, if the relative calibration of the arrays is known, the observed amplitude variation of the received field between arrays provides additional information for source localization: the corresponding processor takes advantage of the fact that the source amplitude spectrum is the same (although unknown) for all arrays. Likewise, if synchronization between arrays is known, phase variation provides localization information. Various multiple-array processors are derived and evaluated in terms of the probability of correct localization from Monte-Carlo analyses. The analysis indicates that, dependent on specific array configurations, significant improvements in source localization performance can be achieved when including relative amplitude and/or phase information in multiple-array processors.

\section{Theory}

Consider measured complex (frequency-domain) acoustic data vectors $\mathbf{d}_{a f}$ at $N_{a}$ arrays (with $H_{a}$ hydrophones each) and $N_{f}$ frequencies due to a source at location $\mathbf{x}=(x, y, z)$. Assuming circularly-symmetric complex Gaussiandistributed errors with variances $\sigma_{f}^{2}$ and complex spectral strengths $\mathbf{S}$ (discussed below), the likelihood function is given by

$$
\begin{aligned}
L(\mathbf{x}, \mathbf{S}, \boldsymbol{\sigma}) & =\prod_{f=1}^{N_{f}} \prod_{a=1}^{N_{a}} \frac{1}{\left(\pi \sigma_{f}^{2}\right)^{H_{a}}} \\
& \exp \left[-\left|\mathbf{d}_{a f}-S e^{i \theta_{a f}} \mathbf{d}_{a f}(\mathbf{x})\right|^{2} / \sigma_{f}^{2}\right]
\end{aligned}
$$

where $\mathbf{d}_{a f}(\mathbf{x})$ represents predicted acoustic fields computed by a numerical propagation model for a unit-amplitude, zero-phase source at location $\mathbf{x}$. The corresponding misfit (negative log-likelihood) function is given by

$$
E(\mathbf{x}, \mathbf{S}, \boldsymbol{\sigma})=\sum_{f=1}^{N_{f}} \sum_{a=1}^{N_{a}}\left|\mathbf{d}_{a f}-S \mathbf{d}_{a f}(\mathbf{x})\right|^{2} / \sigma_{f}^{2}+H_{a} \log _{e} \sigma_{f}^{2} .
$$
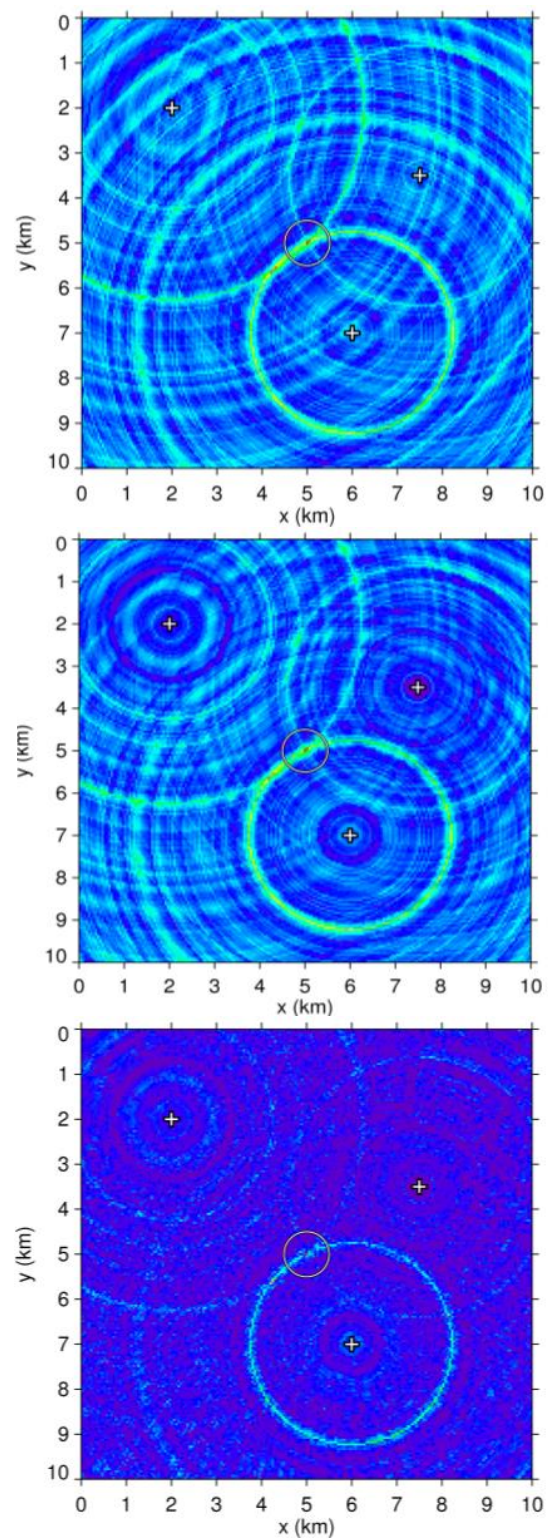

Figure 1: Comparison of horizontal $(x-y)$ ambiguity surfaces (at the optimal source depth) for data from three vertical arrays (crosses) and the incoherent, amplitude-coherent, and coherent processors (top to bottom). The true source position is at $(5,5) \mathrm{km}$, and white circles are centred at the maximum processor value.

Three cases consisting of different assumptions on the relative information available between arrays are considered. The first case assumes that the sensors of each individual array are calibrated and synchronized (have a 
common clock), but that no information on calibration and timing is available between arrays. In this case the unknown spectral strength in Eqs. (1) and (2) is taken to be $S=A_{a f} e^{i \theta_{a f}}$ where $A$ and $\theta$ represent source amplitude and phase, both of which vary with array (subscript $a$ ) and frequency (subscript $f$ ). Maximizing the likelihood over the unknown inter-array amplitude and phase factors as well as the noise variances (which are often unknown) by setting $\partial E / \partial A_{a f}=\partial E / \partial \theta_{a f}=\partial E / \partial \sigma_{f}=0$ leads, after some algebra, to a misfit function (processor)

$$
E(\mathbf{x})=\sum_{f=1}^{N_{f}} \sum_{a=1}^{N_{a}} N_{a} \log _{e}\left[\left|\mathbf{d}_{a f}\right|^{2}-\frac{\left|\mathbf{d}_{a f}(\mathbf{x})^{\dagger} \mathbf{d}_{a f}\right|^{2}}{\left|\mathbf{d}_{a f}(\mathbf{x})\right|^{2}}\right] .
$$

The misfit $E$ in Eq. (3) depends only on the source position $\mathbf{x}$ and the term in square brackets on the right is recognized as the incoherent Bartlett processor which is summed over arrays and frequencies.

The second case consists of relative knowledge of both sensor calibration (amplitude) and synchronization (phase) between arrays. In this case the unknown spectral strength is taken to be $S=A_{f} e^{i \theta_{f}}$ in which amplitude and phase depend on frequency but not array (no subscript $a$ ). Maximizing the likelihood over $A_{f}, \theta_{f}$, and $\sigma_{f}$ leads to a processor

$$
E(\mathbf{x})=\sum_{f=1}^{N_{f}}\left(\sum_{a=1}^{N_{a}} H_{a}\right) \log _{e}\left[\left|\sum_{a=1}^{N_{a}} \mathbf{d}_{a f}\right|^{2}-\frac{\left|\sum_{a=1}^{N_{a}} \mathbf{d}_{a f}(\mathbf{x})^{\dagger} \mathbf{d}_{a f}\right|^{2}}{\left|\sum_{a=1}^{N_{a}} \mathbf{d}_{a f}(\mathbf{x})\right|^{2}}\right],(4)
$$

where the term in square brackets is recognized as the coherent Bartlett processor (over arrays and frequencies).

In many practical settings synchronization between arrays (i.e., a precise common clock) is not available, but the relative array calibrations are known. In this case the unknown spectral strength is taken to be $S=A_{f} e^{i \theta_{a f}}$ in which the relative phase is unknown from array to array (subscript $a$ on $\theta$ ) but the relative amplitude is known. Maximizing the likelihood over $A_{f}, \theta_{a f}$, and $\sigma_{f}$ leads to

$$
E(\mathbf{x})=\sum_{f=1}^{N_{f}}\left(\sum_{a=1}^{N_{a}} H_{a}\right) \log _{e}\left[\sum_{a=1}^{N_{a}}\left|\mathbf{d}_{a f}\right|^{2}-\frac{\left(\sum_{a=1}^{N_{a}}\left|\mathbf{d}_{a f}(\mathbf{x})^{\dagger} \mathbf{d}_{a f}\right|\right)^{2}}{\left|\sum_{a=1}^{N_{a}} \mathbf{d}_{a f}(\mathbf{x})\right|^{2}}\right] .
$$

In Eq. (5) the correlations between modelled and measured fields are summed incoherently (as in Eq. (3)), but the sum of the correlations is normalized by the summed amplitude of the modelled fields (as in Eq. (4)) to preserve relative information on source location contained in the measured field amplitudes from array to array (not included in Eq. (3) where the field at each array is normalized individually). Hence, this processor is refered to as "amplitude coherent."

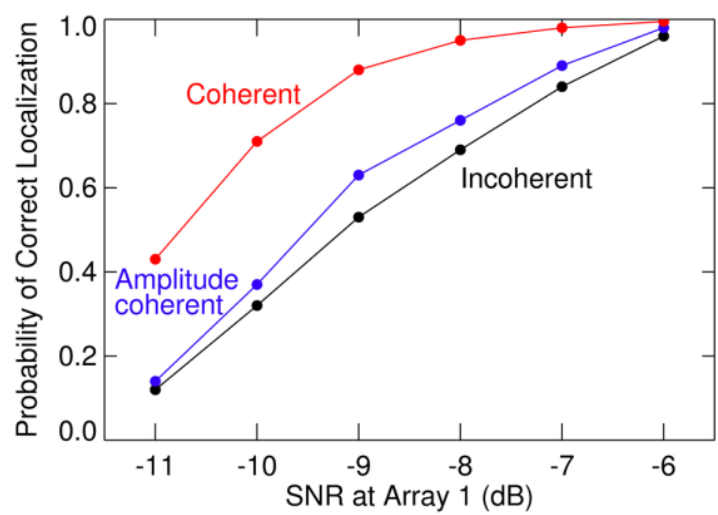

Figure 2: Comparison of probability of correct localization (from 500 random Monte Carlo trials) for the three processors as a function of SNR at Array 1 (upper left in Fig. 1).

\section{Results}

A (simulated) example of ambiguity surfaces for the three processors is shown in Fig. 1 for a source at $(x, y)=(5,5) \mathrm{km}$ and 50-m depth, and three vertical arrays of 24 sensors over a 100-m water column; five frequencies of 50, 100, 200, $300,500 \mathrm{~Hz}$ are considered and the signal-to-noise ratios (SNR) are $-5,-6.5$, and $-8.5 \mathrm{~dB}$ at the three arrays for all frequencies (arrays are numbered clockwise from the upper left in Fig. 1). This figure shows a substantial improvement in the ability to localize the source (relative to background processor values) for the coherent processor (bottom panel) over the incoherent processor (top panel); however, interarray synchroniztaion required for the coherent processor is often unavailable. Figure 1 also shows a smaller but significant improvement over the incoherent processor due to the inclusion of relative calibration information (middle panel), which is frequently available.

The relative performance of the three processors is quantified using a Monte Carlo study in which 500 realizations of noisy data and random source locations were considered for the three processors, with correct localization defined to be within $200 \mathrm{~m}$ in $x$ and $y$ and $10 \mathrm{~m}$ in $z$ of the true location. Figure 2 shows the probability of correct localization for the processors as a function of SNR at Array 1. While Fig. 2 shows the best performance at all SNRs for the coherent processor, the amplitude-coherent processor, which makes use of commonly-available information, is also significantly better than the incoherent processor.

\section{Conclusion}

Three maximum-likelihood processors were derived for matched-field source localization using multiple sensor arrays, differing in the level of knowledge of inter-array calibration and synchronization. The best results are obtained for the coherent processor based on full inter-array information. However, the (new) amplitude-coherent processor based on relative calibration knowledge (commonly available) also out-performs an incoherent processor which incorporates no inter-array information. 